\title{
A New Numerical Calculation Method for Zone Modeling to Predict Smoke Movement in Building Fires
}

\author{
WEICHENG FAN and XINWEI WANG \\ University of Science and Technology of China \\ State Key Laboratory of Fire Science \\ Hefei, Anhui, 230026, P.R. China
}

\begin{abstract}
A new numerical calculation method is presented to predict smoke movement among rooms in building fires using volume conservation and energy conservation instead of the previous mass conservation to modify pressure. Furthermore, in this study network modeling was handled with zone modeling method. Then a new program FZN Ver 3.0 (field, zone, and network modeling) has been developed. In this paper the relevant formula derivation is given. In addition two calculation examples are presented to test the simulation method and program. It is shown that the program could be used to calculate the smoke movement from ignition to any time, and it runs well to predict the smoke movement in building fires.
\end{abstract}

KEYWORDS: building fires, zone modeling, network modeling, modification of pressure, volume conservation, energy conservation.

\section{INTRODUCTION}

Smoke is a very important factor that causes casualties in a building fire. Predictions of smoke movement have drawn great attention in the fire research community ${ }^{[1]-[2]}$. Numerical simulation methods of smoke movement in a building may be generally grouped into four categories, field modeling, zone modeling, network modeling and FZN (fieldzone-network) modeling. Zone modeling method is widely used due to its lower cost and acceptable reliability. In the well-known CFAST method, it is assumed that each room has an uniform pressure for the upper and lower layers. Then by solving a set of ordinary differential equations, smoke spread can be predicted. ${ }^{[3]-[5]}$ In the previous FZN modeling method, the pressure was modified using mass conservation. ${ }^{[6]}$ But its iterative process is 
difficult to converge in the latter period of a building fire. In this paper a new method to modify pressure will be introduced in detail. This method is similar to SIMPLE method solving the N-S equations. The difference is that SIMPLE method uses mass conservation to modify pressure, while this method uses volume conservation or energy conservation.

\section{GOVERNING EQUATIONS}

\section{Governing Equations of Zone Modeling}

As shown in figure 1, there are two rooms : room 1 and room 2 , connected by a vent . The vent can be divided into a given number of cells, point $A$ is assumed to be at the center of a cell. The height of the cell is $\Delta \mathrm{H}$, and its width is $\mathrm{W}$. Through this cell, the mass and energy exchange rates $\Delta \dot{\mathbf{m}}, \Delta \dot{\mathbf{Q}}$ can be calculated as:

$$
\begin{aligned}
& \Delta \dot{\mathrm{m}}=\sqrt{\left|\mathrm{P}_{\mathrm{A} 1}-\mathrm{P}_{\mathrm{A} 2}\right| \cdot 2 \cdot \rho} \cdot \mathrm{C}_{\mathrm{D}} \cdot \Delta \mathrm{H} \cdot \mathrm{W} \\
& \Delta \dot{\mathrm{Q}}=\mathrm{C}_{\mathrm{P}} \cdot \mathrm{T} \cdot \Delta \dot{\mathrm{m}}
\end{aligned}
$$

$\rho, T$ are density and temperature of gas through point $A ; P_{A 1}, P_{A 2}$ are the gas pressures on the two sides of point $A ; C_{P}$ is the specific heat of air at constant pressure, $C_{D}$ is a coefficient indicating vent effects on flow.

The governing equations for each room are:

$$
\begin{aligned}
& \frac{\mathrm{dm}_{\mathrm{i}}}{\mathrm{dt}}=\sum \Delta \dot{\mathrm{m}}_{\mathrm{i}}+\dot{\mathrm{m}}_{\mathrm{i}, \text { source }} \\
& \frac{\mathrm{dQ}}{\mathrm{dt}}=\sum \Delta \dot{\mathrm{Q}}_{\mathrm{i}}+\dot{\mathrm{Q}}_{\mathrm{i} \text { ssource }} \\
& \mathrm{i}=\mathrm{U}, \mathrm{L}
\end{aligned}
$$

$\mathrm{m}, \mathrm{Q}$ are the mass and energy of a gas layer. The subscripts $\mathrm{U}$ and $\mathrm{L}$ refer to the upper layer and lower layer, respectively. The subscript "source" refers to a source term.

Besides, there exist the following relations:

$$
\mathrm{V}_{\mathrm{i}}=\frac{\mathrm{m}_{\mathrm{i}}}{\rho_{\mathrm{i}}}, \quad \mathbf{P}=\rho_{\mathrm{i}} \mathrm{RT}, \mathrm{i}=\mathrm{U}, \mathrm{L}
$$

In CFAST , equations are solved using the method of solving a set of ordinary differential equations, but the solving process is rather complicated. At the early stage of a fire, the time step must be very small. When the building is very large and it includes many rooms, the process of solving is more complicated and the convergence may become difficult. 
In the previous FZN modeling research, pressure was modified with mass conservation method. But its effect is not good because the mass of gas in a room is an implicit parameter, the simulation often diverges. However the sum of the upper and lower gas volume of a room is a constant parameter, therefore it may be more effective to modify pressure with volume conservation.

\section{Pressure Modification Using Volume Conservation}

It is supposed that the pressure on the bottom of a room is $P_{0}$, so the pressure at the height of $\mathrm{h}$ is ,

$$
\mathbf{P}=\mathbf{P}_{0}-\int_{0}^{\mathrm{h}} \rho \mathrm{gdh}
$$

The mass and energy exchange between rooms can be calculated according to the supposed pressure, the temporary parameters can be obtained:

$$
\begin{aligned}
& \mathrm{m}_{\mathrm{i}}=\mathrm{m}_{\mathrm{i}, \text { old }}+\Delta \mathrm{m}_{\mathrm{i}} \\
& \mathrm{Q}_{\mathrm{i}}=\mathrm{Q}_{\mathrm{i}, \text { old }}+\Delta \mathrm{Q}_{\mathrm{i}} \\
& \mathrm{T}_{\mathrm{i}}=\frac{\mathrm{Q}_{\mathrm{i}}}{\mathrm{m}_{\mathrm{i}} \mathrm{C}_{\mathrm{P}}}
\end{aligned}
$$

here the subscript "old" means the calculated value at the last time step.

The density $\rho_{i}$, volume $V_{i}$ can be calculated from equation (5). However the following equation of volume conservation should be satisfied:

$\mathrm{V}_{\mathrm{U}}+\mathrm{V}_{\mathrm{L}}=\mathrm{V}_{\mathrm{R}}$

$V_{R}$ is the volume of the room.

Normally, the calculated volume according to the supposed pressure will not meet the equation of volume conservation, the deviation $\Delta V_{R}$ is:

$$
\Delta \mathrm{V}_{\mathrm{R}}=\mathrm{V}_{\mathrm{U}}+\mathrm{V}_{\mathrm{L}}-\mathrm{V}_{\mathrm{R}}
$$

In order to eliminate the deviation, a modification $\Delta \mathrm{P}_{0}$ should be added to the supposed pressure, the mass exchange $\Delta\left(\Delta \mathrm{m}_{\mathrm{i}}\right)$ and volume variation $\Delta \mathrm{V}_{\mathrm{i}}$ caused by this pressure modification are as the following: 
$\Delta\left(\Delta m_{i}\right)=\sum_{i} \frac{\sqrt{\rho}}{\sqrt{2\left|P_{A 1}-P_{A 2}\right|}}\left(-\Delta P_{01}+\Delta P_{02}\right) \cdot \Delta H \cdot C_{D} \cdot W \cdot \Delta t$
$\Delta V_{i}=\frac{\Delta\left(\Delta m_{i}\right)}{\rho_{i}}=\sum_{i} \frac{\sqrt{\rho}}{\rho_{i} \sqrt{2\left|P_{A 1}-P_{A 2}\right|}}\left(-\Delta P_{01}+\Delta P_{02}\right) \cdot \Delta H \cdot C_{D} \cdot W \cdot \Delta t$

Subscripts 01,02 refer to room 1 and room 2.

$\Delta \mathrm{V}_{\mathrm{U}}, \Delta \mathrm{V}_{\mathrm{L}}$ must meet the volume conservation equation (10):

$\mathrm{V}_{\mathrm{U}}+\Delta \mathrm{V}_{\mathrm{U}}+\mathrm{V}_{\mathrm{L}}+\Delta \mathrm{V}_{\mathrm{L}}=\mathrm{V}_{\mathrm{R}}$

Then combining with equation (11), we can obtain:

$\Delta \mathrm{V}_{\mathrm{U}}+\Delta \mathrm{V}_{\mathrm{L}}=-\Delta \mathrm{V}_{\mathrm{R}}$

The above $\Delta\left(\Delta \mathrm{m}_{\mathrm{i}}\right)$ is calculated for room 1 .

For room 1, now let:

$A_{01}=\frac{\sqrt{2}}{2}\left\{\sum_{U} \frac{\sqrt{\rho}}{\rho_{U} \sqrt{\left|P_{A 1}-P_{A 2}\right|}}+\sum_{L} \frac{\sqrt{\rho}}{\rho_{L} \sqrt{\left|P_{A 1}-P_{A 2}\right|}}\right\} \cdot \Delta H \cdot C_{D} \cdot W \cdot \Delta t$

According to equations 12-13, we can obtain:

$A_{02}=A_{01}$

Pressure modification equation of room 1 can be obtained:

$A_{01} \cdot \Delta P_{01}-A_{02} \Delta P_{02}=\Delta V_{R}$

That is:

$A_{01} \Delta P_{01}=A_{02} \Delta P_{02}+\Delta V_{R}$

Generally one room could be adjacent to six rooms, that is, top, bottom ,north, south, east and west rooms. If there exist several vents between the room and its adjacent rooms, the 
relevant coefficients of the pressure modification equations can also be obtained:

$A_{i}=\frac{\sqrt{2}}{2}\left\{\sum_{U} \frac{\sqrt{\rho}}{\rho_{U} \sqrt{\left|P_{A 1}-P_{A 2}\right|}}+\sum_{L} \frac{\sqrt{\rho}}{\rho_{L} \sqrt{\left|P_{A 1}-P_{A 2}\right|}}\right\} \cdot \Delta H \cdot C_{D} \cdot W \cdot \Delta t$

$\mathrm{i}=\mathrm{T}, \mathrm{B}, \mathrm{N}, \mathrm{S}, \mathrm{E}, \mathrm{W}$

$A_{P}=\sum A_{i}$

Then the pressure modification equation will be:

$A_{P} \cdot \Delta P=\sum_{i} A_{i} \Delta P_{i}+\Delta V_{R}$

$\mathrm{i}=\mathrm{T}, \mathrm{B}, \mathrm{N}, \mathrm{S}, \mathrm{E}, \mathrm{W}$

\section{Pressure Modification Using Energy Conservation}

During the process of pressure modification using volume conservation, implicit method is used for all the parameters of smoke such as density, temperature, but the entrainment induced by smoke movement can not be calculated well, especially in the latter period of a fire, so this method needs to be improved. When using energy conservation to modify pressure, the implicit method is only used to calculate pressure, other parameters can be calculated by explicit methods, thus the solving process can be improved.

From equations (5) and (10), we can obtain the following relations:

$\frac{\mathbf{m}_{U} \mathbf{R T}_{\mathrm{U}}}{\mathbf{P}}+\frac{\mathbf{m}_{\mathrm{L}} \mathrm{RT}_{\mathrm{L}}}{\mathrm{P}}=\mathrm{V}_{\mathrm{R}}$

$m_{U} T_{U} C_{P}+m_{L} T_{L} C_{P}=\frac{V_{R} P C_{P}}{R}=$ Const

From the above equation we can obtain a conclusion that the energy of gas in a room is constant. Through a similar process to above derivation for volume conservation, we can obtain the coefficients of pressure modification using energy conservation:

$A_{i}=\frac{\sqrt{2}}{2}\left\{\sum_{U} \frac{T_{U} \sqrt{\rho}}{\sqrt{\left|P_{A 1}-P_{A 2}\right|}}+\sum_{L} \frac{T_{L} \sqrt{\rho}}{\sqrt{\left|P_{A 1}-P_{A 2}\right|}}\right\} \cdot \Delta H \cdot C_{D} \cdot W \cdot \Delta t$

$\mathrm{i}=\mathrm{T}, \mathbf{B}, \mathbf{N}, \mathrm{S}, \mathrm{E}, \mathrm{W}$

$A_{P}=\sum A_{i}$ 
The pressure modification equation is:

$$
\begin{aligned}
& \mathrm{A}_{\mathrm{P}} \cdot \Delta \mathbf{P}=\sum_{\mathrm{i}} \mathrm{A}_{\mathrm{i}} \Delta \mathrm{P}_{\mathrm{i}}+\Delta \mathbf{Q}_{\mathrm{R}} \\
& \Delta Q_{R}=\left(\sum_{U} \Delta \dot{Q}_{U}+\sum_{L} \Delta \dot{Q}_{L}\right) \cdot \Delta t+\dot{Q}_{\text {Sourse }} \cdot \Delta t
\end{aligned}
$$

$\mathrm{i}=\mathrm{T}, \mathrm{B}, \mathrm{N}, \mathrm{S}, \mathrm{E}, \mathrm{W}$

\section{Network Modeling Using Zone Modeling Method}

During the FZN simulation of smoke movement, field modeling is used in the fire room, zone modeling is used in the rooms adjacent to the fire room, network modeling is used in the rooms far from the fire room. For network modeling, it is often necessary to write a new program to be fitted into the FZN model. In fact, network modeling can be handled with zone modeling method with each room only having one layer of gas. Then a similar method to zone modeling can be used to calculate the mass and energy exchange:

$$
\begin{aligned}
& T=\frac{Q_{U}+Q_{L}}{\left(m_{U}+m_{L}\right) C_{P}} \\
& Q_{L}=Q_{U}+Q_{L} \\
& m_{L}=m_{U}+m_{L}
\end{aligned}
$$

Then let $Q_{U}=0, m_{U}=0$, we can start the calculation of a new time step.

\section{SOLUTION PROCEDURE}

Solution procedure for pressure modification equation using energy conservation is as follows:

1. Take $P_{0}$, the pressure at time $t$, as the initial value at time $t+\Delta t$, then calculate the $\Delta \mathrm{m}_{\mathrm{i}}, \Delta \mathbf{Q}_{\mathrm{i}}$ and other parameters.

2. Calculate $\Delta Q_{R}$ and the coefficients $A_{i}$ ( $\left.i=T, B, N, S, W, E, P\right)$, and solve the pressure modification equation by TDMA method. Then calculate $\Delta P$, and let $P_{0}=P_{0}+\Delta P$.

3. Based on the new $P_{0}$, go back to step 1 to continue the calculation until $\Delta Q_{R}$, the source item of pressure modification equation, is less than a prescribed small quantity. 
When calculating the pressure, it is often helpful to use under-relaxation method: $\mathbf{P}_{0}=\mathbf{P}_{0}+\omega \Delta \mathbf{P}$, the relaxing factor $\omega$ is $0.001 \sim 0.1$

\section{EXAMPLES OF CALCULATION}

The following examples have been studied to verify the feasibility of pressure modification with energy conservation and the handling method of network modeling.

Example 1: As shown in figure 1, there are two identical rooms connected by a vent.

Size of each room: Length $\times$ Width $\times$ Height : $5.0 \times 4.0 \times 3.5\left(\mathrm{~m}^{3}\right)$

Size of the vent: Width $\times$ Height : $\quad 2.0 \times 2.5\left(\mathrm{~m}^{2}\right)$

Initial conditions: the smoke layer in room 1 is $2.2 \mathrm{~m}$ thick, its temperature is $450 \mathrm{~K}$; the temperature of the lower layer is $288.15 \mathrm{~K}$.

The calculation results of example one are shown in figures 2, 3 and 4.

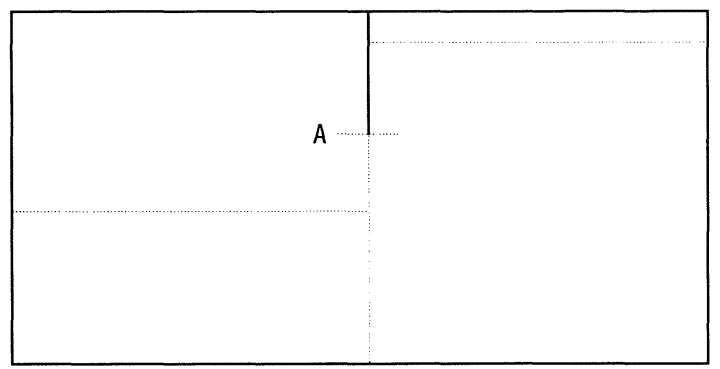

Fig. 1 Vertical-section schetch of the building for the first example of calculation

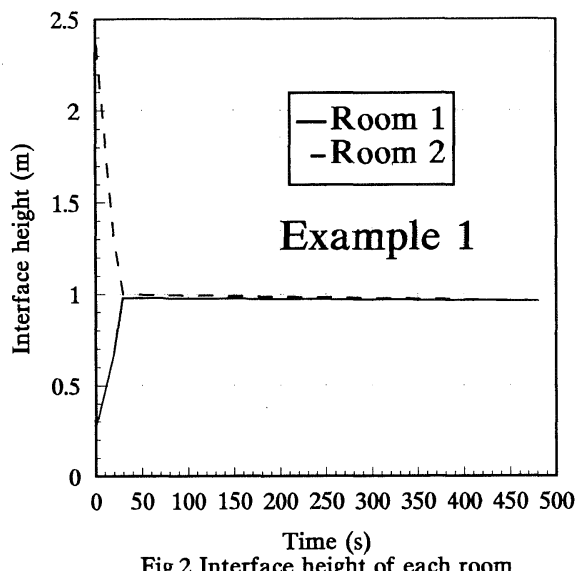

Fig.2 Interface height of each room 


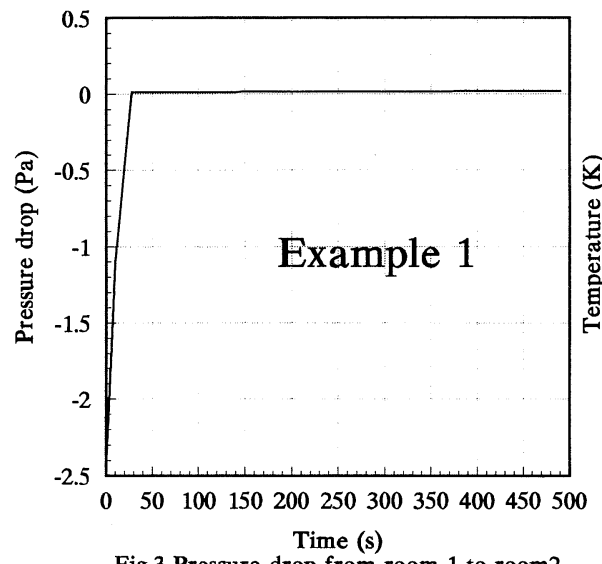

Fig.3 Pressure drop from room 1 to room2

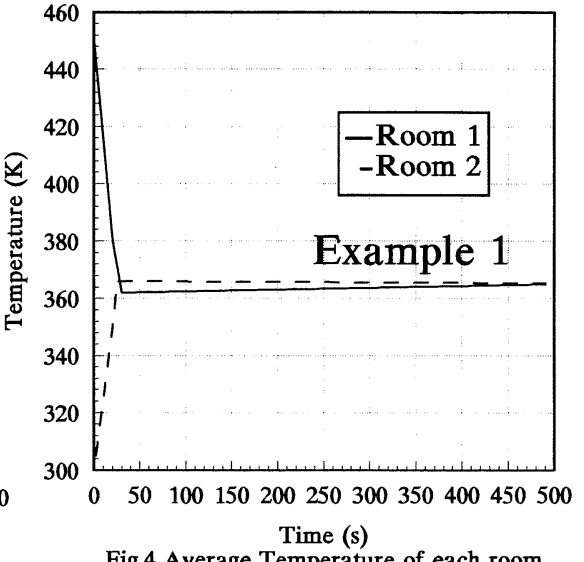

Fig.4 Average Temperature of each room

Example 2: The building for this calculation example is shown in figure 5.

Room size: $\quad$ Length $\times$ Width $\times$ Height $(\mathrm{m})$

Fire room: $\quad 4.22 \times 3.35 \times 2.44$

Corridor: $\quad 18.97 \times 2.41 \times 2.44$

Lobby: $\quad 3.0 \times 15.4 \times 2.44$

Vent size: Width $\times$ Height $(\mathrm{m})$

V1: $\quad 1.07 \times 2.03$

V2: $\quad 0.94 \times 1.5$

V3: $\quad 1.25 \times 2.01$

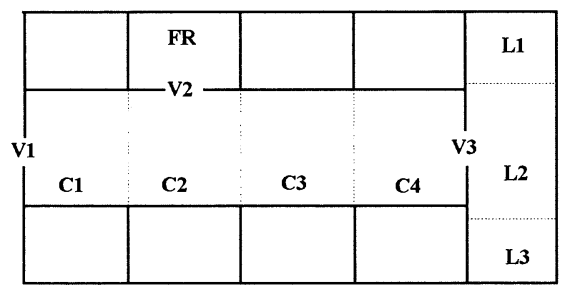

Fig. 5 Horizontal-section schetch of the building for the second example of calculation 
In the fire room (denoted with FR in figure 5), field modeling is used; the corridor is divided into four parts, C1, C2, C3 and C4, and for each part, zone modeling is used; the lobby is divided into three parts, L1, L2 and L3, and for each part, the network modeling is used.

The boundary conditions of field modeling: there is a heat source on the floor $(0.5 \mathrm{~m} \times$ $0.5 \mathrm{~m}$ ), the temperature of the heat source is $1380 \mathrm{~K}$, the mass release rate is $0.091 \mathrm{~kg} / \mathrm{s}$ 。 Limited by the length of the paper, only a part of results are shown.

Firstly, we used mass conservation to modify the pressure. It was found that the relaxation factor must be very small in the calculation. But even so, after about 100 seconds, the residue became so large that the calculation would be difficult to continue. However when using the energy conservation to modify the pressure, the speed of convergence is found to be much greater than that using mass conservation, and we can continue the calculation as long as required.

The calculation results of example two are shown in figures $6,7,8,9$ and 10 .

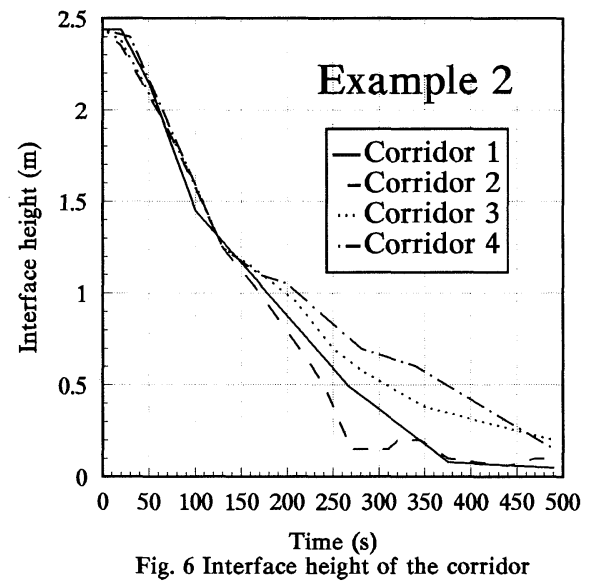




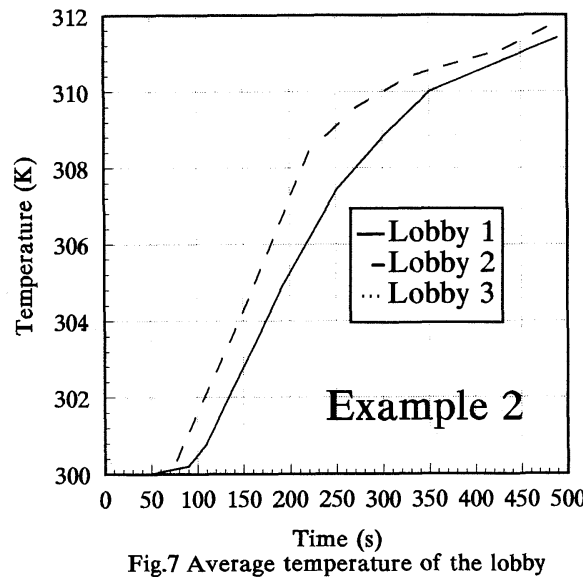

Fig.7 Average temperature of the lobby

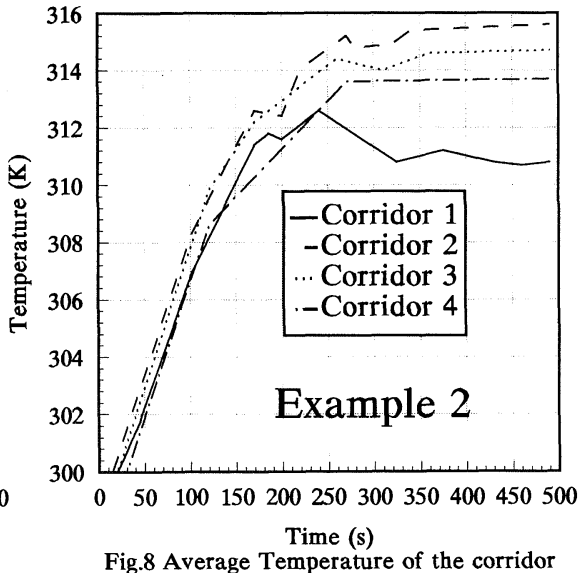

Fig.8 Average Temperature of the corridor

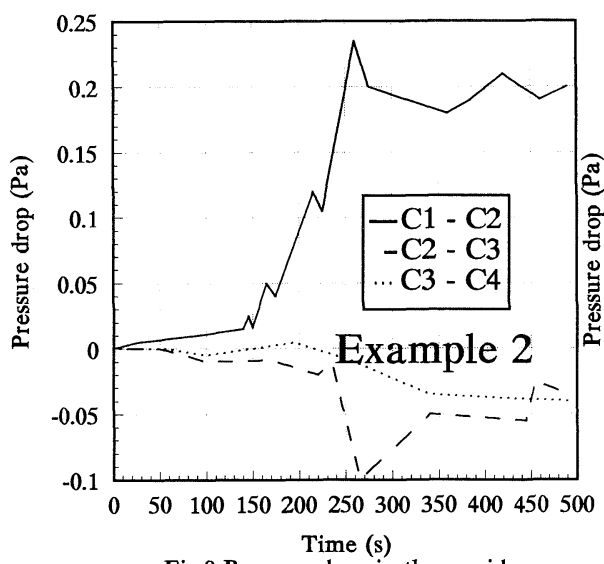

Fig.9 Pressure drop in the corridor

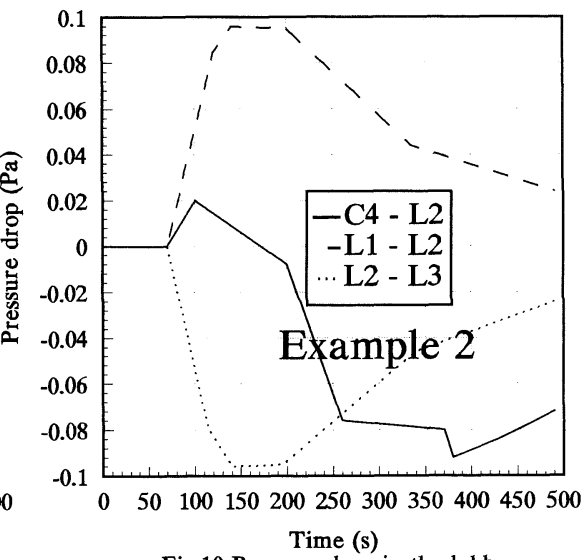

Fig.10 Pressure drop in the lobby 


\section{CONCLUSION}

By using energy conservation to modify pressure and zone modeling method to simulate the network modeling, a new numerical calculation method and program has been developed. From the calculation it is found that the program runs well, and the results are reasonable. Further work will be conducted to compare simulation results with experimental data.

\section{ACKNOWLEDGMENTS}

The authors would like to thank the State Science and Technology Commission of China (SSTCC) and National Natural Science Foundation of China (NNSFC) for their support.

\section{NOTATION}

Variables

A-modification coefficient

$\mathbf{C}_{\mathbf{P}}$-specific heat at constant pressure m-mass

Q-heat

T-temperature

W-width

$\Delta$ H-height

$\Delta \mathbf{P}$-pressure difference

$\Delta \mathbf{t}$-time step size

$\Delta(\Delta \mathrm{m})$-mass exchange caused by modification

\section{Subscripts}

01-room 1

U-upper layer

R-room

B-bottom room

S-south room

W-west room
$\mathbf{C}_{\mathbf{D} \text {-vent flow coefficient }}$

g-gravitational acceleration

P-pressure

R-general gas constant

V-volume

$\rho$-density

$\Delta \mathbf{m}$-mass exchange rate

$\Delta \mathbf{Q}$-heat exchange rate

$\Delta \mathbf{V}$-volume deviation due to modification 02-room 2

L-lower layer

T-top room

N-north room

E-east room 


\section{REFERENCES}

1. Fan, W., Li, S., "Fire Research in China", Proceedings of the Fourth International Symposium on Fire Safety Science, 27-45, Ottawa, 1994.

2. Fan, W., Wang, Q., Zhang, R., Huo, R., An Introduction to Fire Science, 403-425, Hubei Science and Technology, Wuhan, China, 1993. (in Chinese)

3. Jones, W.W., Forney, G.P., "Improvement in Predicting Smoke Movement in Compartment Structures", Fire Safety Journal, 21, 269, 1993.

4. Peacock, R.D., Jones, W.W. and Bukowski, R.W., "Verification of a Model of Fire and Smoke Transport", Fire Safety Journal, 21:2, 89-129, 1993.

5. Fu, Z., Fan, W., "A Zone-type Model for a Building Fire and Its Sensitivity Analysis", Fire and Materials, 20:5, 215-224, 1996.

6. Fan, W., Yan, Z., Huo, R., "A Combined Field-Zone Model for Compartment Fire", Proceedings of the First Asian Conference on Fire Science and Technology, 39-49, Hefei, 1992. 